Association for Information Systems

AIS Electronic Library (AISeL)

Wirtschaftsinformatik 2021 Proceedings

Track 4: Creating value through digital

innovation in health care

\title{
Let's Get Engaged: On the Evidence of Patient Engagement Tools and Their Integration in Patient Pathways
}

\author{
Emily Hickmann \\ Technische Universität Dresden \\ Peggy Richter \\ Technische Universität Dresden \\ Hannes Schlieter \\ Technische Universität Dresden
}

Follow this and additional works at: https://aisel.aisnet.org/wi2021

Hickmann, Emily; Richter, Peggy; and Schlieter, Hannes, "Let's Get Engaged: On the Evidence of Patient Engagement Tools and Their Integration in Patient Pathways" (2021). Wirtschaftsinformatik 2021

Proceedings. 10.

https://aisel.aisnet.org/wi2021/WCreating/Track04/10

This material is brought to you by the Wirtschaftsinformatik at AIS Electronic Library (AISeL). It has been accepted for inclusion in Wirtschaftsinformatik 2021 Proceedings by an authorized administrator of AIS Electronic Library (AISeL). For more information, please contact elibrary@aisnet.org. 


\title{
Let's Get Engaged: On the Evidence of Patient Engagement Tools and Their Integration in Patient Pathways
}

\author{
Emily Hickmann ${ }^{1}$, Peggy Richter ${ }^{1}$, and Hannes Schlieter ${ }^{1}$ \\ ${ }^{1}$ Technische Universität Dresden, Chair of Wirtschaftsinformatik, esp. Systems Development, \\ Dresden, Germany \\ \{emily.hickmann,peggy.richter2,hannes.schlieter\}@tu-dresden.de
}

\begin{abstract}
Patient pathways are a means to structure the care process for patients with complex and long-term diseases in integrated care networks. Simultaneously, they have a stronger emphasis on the patient perspective and engagement than related pathway concepts. Still, there are no common mechanisms for patient engagement concepts in patient pathway models. This paper therefore explores the state-of-the-art of patient engagement tools as well as evidence on their effectivity and feasibility, picking the Option Grid, the Patient Diary, and the Question Prompt Sheet (QPS) as representative examples. Based on this, we propose recommendations for the representation of such tools in patient pathway models and demonstrate them with the application of the QPS in a colorectal cancer patient pathway. To conclude, the evidence on patient engagement tools is still diverse but promising. Anchoring successful tools in patient pathways holds the potential to support their broader application and enhance individualized care.
\end{abstract}

Keywords: patient engagement, patient pathway, shared decision-making, literature review

\section{Introduction}

Current challenges in the health care sector, including sectoral boundaries, the financing system and demographic changes, result in an increased need for a transparent and well-organized coordination of patients through their individual care processes. At the same time, an efficient distribution of resources has to be ensured. The recreation of processes is a central strategy to combat these challenges, as a welldesigned process can for instance promote continuity of care, ensure an efficient resource allocation and support the decision-making process [1]. In the health care sector, a common tool used to design processes is the pathway.

In medicine, there is no standardized definition for the term pathway. Küttner and Roeder (2007) [2] describe three main components of pathways that seem to be prominent in all definitions: They refer to a specific patient group, are used by an interprofessional treatment team and define a diagnostic and therapeutic action corridor 
[2]. A major concern regarding pathways in medicine is that they could foster depersonalisation, as they may be based too heavily on the requirements of an average patient, leaving diminutive space for individual needs and decisions. Even though a main aim of pathways is to reduce variations and therefore guarantee all patients the same level of a high-quality treatment, they also have the potential to foster individualization. These concepts may seem contrary at first, however a well-designed pathway must be flexible enough to be personalized to individual cases in a standardized manner [3]. For example, a pathway can incorporate steps where the patients are systematically asked for feedback or input on the respective health issue. This information could then decide the further route that is taken in the pathway. It therefore needs to enable the users to navigate patients through different options and stages, depending on the individual decision-making process [4]. Compared to other pathway approaches, patient pathways have a very prominent focus on individualization [5]. Therefore, the concepts of patient engagement and shared decision-making (SDM) need to have a central part in the design and implementation of the pathway process.

There is no widely accepted definition of the term patient engagement, however a comprehensive definition by Higgins et al. (2017) defines it as "the desire and capability to actively choose to participate in care in a way uniquely appropriate to the individual, in cooperation with a healthcare provider or institution, for the purpose of maximizing outcomes or improving experiences of care" [6]. An important aspect of this definition is a patient's capability to participate. In order to be capable, patients must acquire the necessary knowledge to decide, which is an integral part of patient empowerment. Other characteristics include capacity building, gaining control over the situation, motivation, self-care and trust [7]. SDM is an integral part of engaging patients into their health care process. It implies an active engagement of the patient and the physician in the decision-making process by sharing information and personal values [7-8].

Actively engaging patients yields multiple benefits for all stakeholders along the care process. Engaged patients have a better awareness and understanding of their condition, leading to an enhanced communication with their health care professionals [9]. As a result, compliance is fostered and the health status improves. Different authors [9-11] agree that patient engagement has the potential to reduce health care costs and enhance a more appropriate and effective usage of resources. The quality of health care delivery is enhanced further, as less treatment errors tend to occur when patients are engaged in the process [9-11]. When combining the concepts of patient pathways and patient engagement a higher quality of care can be guaranteed throughout the health care process. Patient pathways will become more individualized, therefore putting more emphasis on patient's individual needs. Simultaneously, patient engagement concepts are not yet represented in patient pathways to support these aims.

Therefore, the research objective of this paper is to explore how patient engagement tools (i.e. an item that supports the user in enhancing patient engagement, similar to an instrument or a utensil) can be integrated into patient pathways. In order to do this, diverse patient engagement tools will be analyzed and opportunities for their practical implementation into patient pathways will be shown. Two research questions (RQ) are 
to be answered: RQ1: What is the evidence for the effectivity and feasibility of patient engagement tools? The effectivity of the respective tool refers to the extent to which the goals, or characteristics of patient engagement are enhanced through its implementation or usage. Feasibility refers to how practical and acceptable the tool is for all stakeholders involved in the process. RQ2: How can patient engagement tools be used in patient pathways?

Accordingly, the remainder of this article is structured as follows: The used method of a literature review is described in section 2. The review results are given in section 3. In total, three out of nine evaluated tools are presented in this paper (selection criteria explained in 2.1). These are the Option Grid, the Patient Diary, and the Question Prompt Sheet (QPS), which are described in subsection 3.1 (referring to answering RQ1). In section 3.2, a representation form for the utilization of patient engagement tools in patient pathways is proposed (referring to answering RQ2). For demonstration purposes, the representation of an engagement tool in a colorectal cancer patient pathway is used as an example. The paper closes with a conclusion and discussion in section 4 .

\section{Method}

\subsection{Preliminary Study on Patient Engagement Tools}

A preliminary study, with the objective to present the current state-of-the-art on tools to engage patients into their health care process was conducted. For this purpose, a literature review in the scientific database PubMed was performed in November 2019. The search string consisted of alternative terms for "patient engagement" in combination with the terms "method", "tool", "aid", "instrument", "strategy" or "implementation". In total 772 articles were identified. From 228 full-text articles that were assessed for eligibility, 53 records were included in the final preliminary study. A study was included if any kind of tool (including the alternative terms used above) was used to involve patients in their own treatment or care. Extraneous topics, such as training programmes or challenges of patient engagement were excluded. The results are a mixture of specific tools, but also diverse strategies that either the physician or the patient can utilize to enhance patient engagement. When only considering the concrete, practical tools (strategies were generally too concrete for a broad evaluation) the following nine could be distinguished: Adaptive Conjoint Analysis (ACA), Best Case/ Worst Case (BC/WC), Decision Box, Option Grid, Patient Empowerment Tool (PET), Patient Diary, Patient Portals, Question Prompt Sheets and the Roulette Wheel. These were evaluated and due to space limitations only three of them were selected for a detailed result presentation in this paper. The Option Grid, Patient Diary and Question Prompt Sheet were chosen for this purpose, as they are intensively considered in literature, can be used in diverse health settings and at different points in time along the patient pathway (i.e. diagnosis, treatment, rehabilitation). The results on the other tools are summarized only shortly in section 3.1. 


\subsection{Literature Review on the Evidence of Patient Engagement Tools}

To answer RQ1, a literature review following the guidelines proposed by Rowley and Slack (2004) [12] was conducted. In the first step, a quick scan on the respective tool was performed, in order to gain a general understanding about its operating mode and possible alternative terms that are used in literature. This information was used to create the search string for the tools, which is depicted in Table 1. A separate search was conducted for each tool and the search string was partially adapted depending on the functionalities of the respective database. Furthermore, the search string for patient portals and personal health records was adapted to include an outcome component to specify the results. This was not necessary for other tools, due to their low prominence in literature.

Table 1. Search string used for literature review

\begin{tabular}{|c|c|c|}
\hline $\begin{array}{c}\text { Patient OR Patients } \\
\text { (PubMed) } \\
\text { Patient\$ (Web of Science) }\end{array}$ & $\begin{array}{l}\text { AND (PubMed) } \\
\text { NEAR/4 (Web of } \\
\text { Science) }\end{array}$ & $\begin{array}{c}\text { Empower* OR Engag* OR } \\
\text { Involv* }\end{array}$ \\
\hline \multicolumn{3}{|c|}{ OR } \\
\hline \multicolumn{3}{|c|}{ Shared decision making } \\
\hline \multicolumn{3}{|c|}{ OR } \\
\hline \multicolumn{3}{|c|}{ Patient participation [MeSH Term] (PubMed only) } \\
\hline \multicolumn{3}{|c|}{ AND } \\
\hline \multicolumn{3}{|c|}{ Adaptive Conjoint Analysis } \\
\hline \multicolumn{3}{|c|}{ Best Case/ Worst Case } \\
\hline \multicolumn{3}{|c|}{ Decision Box* } \\
\hline \multicolumn{3}{|c|}{$\begin{array}{c}\text { Option Grid* (PubMed) } \\
\text { Option Grid\$ (Web of Science) }\end{array}$} \\
\hline \multicolumn{3}{|c|}{ Patient Empowerment Tool } \\
\hline \multicolumn{3}{|c|}{ Diary OR Diaries (PubMed) } \\
\hline \multicolumn{3}{|c|}{$\begin{array}{c}\text { (Personal health record* OR Patient portal*) AND (Outcome* OR Effect* OR } \\
\text { Consequence*) (PubMed) } \\
\text { (Personal health record\$ OR Patient portal\$) NEAR/6 (Outcome* OR Effect* OR } \\
\text { Consequence*) (Web of Science) }\end{array}$} \\
\hline \multicolumn{3}{|c|}{$\begin{array}{c}\text { Question Prompt Sheet* (Pub Med) } \\
\text { Question Prompt Sheet\$(Web of Science) }\end{array}$} \\
\hline \multicolumn{3}{|c|}{$\begin{array}{l}\text { Roulette wheel* OR Dart board* OR Pie chart* (PubMed) } \\
\text { Roulette wheel\$ OR Dart board\$ OR Pie chart\$ (Web of Science) }\end{array}$} \\
\hline
\end{tabular}

During the literature selection process, any record that addressed effectivity or feasibility of patient engagement, as defined in section 1, was included. Publications focusing on extraneous topics were excluded. For example, articles not referring to the tool, as described in section 3.1, were excluded. This was, however, seldomly the case, because the individual search string already contained the specific name of the tool. The literature selection process is summarized in Table 2. 
Table 2. Literature selection process

\begin{tabular}{|c|c|c|c|c|c|}
\hline ACA & 12 & 9 & 9 & 8 & 7 \\
\hline $\mathrm{BC} / \mathrm{WC}$ & 9 & 5 & 5 & 4 & 4 \\
\hline Decision Box & 15 & 8 & 8 & 6 & 2 \\
\hline Option Grid & 32 & 21 & 21 & 13 & 9 \\
\hline PET & 2 & 1 & 1 & 1 & 1 \\
\hline Patient diary & 53 & 51 & 51 & 12 & 6 \\
\hline Patient portal & 73 & 70 & 70 & 26 & 14 \\
\hline QPS & 13 & 10 & 10 & 6 & 4 \\
\hline Roulette Wheel & 4 & 3 & 3 & 3 & 3 \\
\hline
\end{tabular}

After completing this process, the information retrieved from the review process was structured and is summarized in section 3 . As the records selected have very different study designs and therefore levels of reliability, the Oxford scale of evidence was used to put the obtained information into context. The evidence level (EL) of each included study is noted in brackets behind the references of the study and an overview is given in Table 3. Levels could be graded down on basis of study quality, imprecision, indirectness, because of inconsistency between studies or because the absolute effect size was very small. Studies could be graded up if there was a large effect size. Systematic reviews were generally assessed as better than individual studies [13]. They will also be referred to in the individual summaries for each tool, when answering RQ1.

Table 3. Number of sources used assessed with the oxford scale of evidence

\begin{tabular}{|l|c|c|c|c|c|c|c|c|c|}
\hline EL & ACA & $\begin{array}{l}\text { BC/ } \\
\text { WC }\end{array}$ & $\begin{array}{l}\text { Decision } \\
\text { Box }\end{array}$ & $\begin{array}{l}\text { Option } \\
\text { Grid }\end{array}$ & $\begin{array}{l}\text { Patient } \\
\text { Diary }\end{array}$ & $\begin{array}{l}\text { Patient } \\
\text { Portal }\end{array}$ & PET & QPS & $\begin{array}{l}\text { Roulette } \\
\text { Wheel }\end{array}$ \\
\hline I & 0 & 0 & 0 & 0 & 1 & 1 & 0 & 0 & 0 \\
\hline II & 1 & 0 & 0 & 0 & 1 & 3 & 0 & 3 & 0 \\
\hline III & 2 & 0 & 0 & 2 & 1 & 5 & 0 & 0 & 1 \\
\hline IV & 4 & 4 & 2 & 6 & 2 & 5 & 1 & 1 & 1 \\
\hline V & 0 & 0 & 0 & 1 & 1 & 0 & 0 & 0 & 1 \\
\hline
\end{tabular}

I: $\quad$ Systematic review of randomized trials or n-of-1 trials

II: $\quad$ Randomized trial or observational study with dramatic effect

III: Non-randomized controlled cohort/ follow-up study

IV: Case-series, case-control studies, or historically controlled studies

$\mathrm{V}$ : Mechanism-based reasoning 


\section{$3 \quad$ Results}

\subsection{Evidence-based Patient Engagement Tools}

Option Grids. An Option Grid is a one- to maximum three-page summary of all available healthcare options for a specific treatment decision. The information is categorized in form of patients most frequently asked questions when considering different treatment options. For example, likely outcomes, risks and benefits are commonly discussed. Providers can also choose which options they want to present to the patient and can customize the grid with patient-specific data [14-15].

Three of the included studies were conducted on Option Grids for knee osteoarthritis. During the first study, a step-wedged trial with a population of older patients (with lower than average health literacy), the Option Grid led to higher knowledge levels of the patients about the osteoarthritis and its treatment possibilities. Furthermore, an increased readiness to decide for one of the options and an overall improvement of the SDM levels could be observed. This enhanced patient engagement was achieved without prolonging the duration of the encounters [16] (EL: III). During the second study, clinician interviews were performed before and after adoption of the knee osteoarthritis Option Grid. After initial concerns before adoption, the usage of the tool was generally seen as acceptable and helpful for the communication process during the patient encounter, while simultaneously helping clinicians take on a more neutral position. Additionally, they experienced that the patients had a more active role, asking more questions during the consultation [17] (EL: IV). In the third study by Kinsey et al. (2017) interviews with patients using the Option Grid during consultation and a control group were performed. The patients in the intervention group showed an increased awareness of the different treatment options, while the patients in the control group were less clear about the fact that different treatment options had been discussed. The physicians working with the control group also seemed to focus the discussion on risks and benefits concerning the (for them) most likely option. Acceptability of the tool for patients was rated as high. Most patients in the intervention group felt more involved in the decision-making process, however both groups felt that they had finally made their own treatment decision [18] (EL: III).

In a further study by Smith et al. (2019) an Option Grid for knee replacement surgery was evaluated. The Option Grid made patients feel better informed and provided them with a starting point for further individual research. This is an important aspect for the development of patient engagement [19] (EL: IV).

Two studies focused on Option Grids for breast cancer. Both studies concluded that the Option Grid was acceptable and feasible for facilitating patient involvement and for improving the perceived understanding of patients. The study by Hahlweg et al. (2019) (EL: IV) highlighted the importance of training physicians on the usage of the Option Grid in order to promote acceptance [20-21] (EL: IV). In a further study, an Option Grid for the usage of antipsychotic medication was evaluated positively. In interviews the tool was perceived as usable, context appropriate and feasible in psychiatric consultations by patients, psychiatrists, family members and administrators [22] (EL: IV). This is supported by the opinion of a general practitioner and professor of primary 
health care, who concludes that the information in Option Grids is presented in a format that allows both reflection and dialogue. In contrast to other SDM-tools the physician also sees the benefit in the simplicity of Option Grids, stating that "neither the patient nor the clinician needs to be a geek to use them" [23] (EL: V).

Only one study was found, in which an Option Grid did not have an influence on the degree of SDM. This was a pre-post intervention study by Scalia et al. (2018), in which over a time period of three months the Option Grid tool was used for diverse conditions in a clinical setting [24] (EL: IV).

Patient Diary. A Patient Diary is a simple tool that can be used by patients for selfmonitoring. For example, symptoms, body weight, blood pressure or activities can be recorded and, when necessary, presented to health care providers [25].

Several records evaluated the feasibility of Patient Diaries. For example, feasibility and acceptance of internet-based and telephone-based diaries were tested in a study by Cherenack et al. (2016) amongst a population of 61 young HIV-infected men. Diary data and qualitative interviews showed that the internet diaries were preferred by $92 \%$ of the population with a completion rate of $78 \%$ over a 66-day measure. Generally, keeping the diaries was described as promoting self-reflection and behavior tracking [26] (EL: IV).

During a study with 393 rural patients, who recorded symptoms on heart failure, it was found that participants actively using a Patient Diary lived longer. For example, patients with a "very high" diary usage, were $39 \%$ less likely to die due to heart failure compared to patients using no diary [27] (EL: III). Using the diary is closely connected to self-management skills and treatment adherence, due to which these results can be partially explained.

Hodge (2013), a family physician and clinical instructor explains that Patient Diaries have a series of advantages. These include that keeping diaries gives patients a sense of control, therefore engaging them more into the treatment process. Furthermore, in terms of feasibility, it takes physicians less time to review the one-page diary than to verbally interview a patient for the same information [28] (EL: V). This opinion is supported by a study of Himes et al. (2016). It was found that self-management programs that include diaries, compared to those that do not, are associated with a higher disease control, enhanced life quality and fewer hospital visits [29] (EL: IV).

In direct contrast to this, are the results of the study by Schmidt et al. (2015). A trial comparing length of hospital stay and quality of life (one year after hospitalization) in a group of 652 patients concluded that the diary did not have an effect on these aspects. Participants were randomly assigned to receive either standard care or an information booklet and a diary. Their mean age of the patients was 72 years. Patient empowerment through booklet and diary did, however, have a positive influence on patient's shortterm well-being, such as postoperative pain [30] (EL: II). Also, a systematic review by Ullman et al. (2014) concludes that there is minimal evidence from randomized controlled trials that Patient Diaries do any benefit or harm. This review was set in the context of patients in the intensive care unit [31] (EL: I). 
Question Prompt Sheet. QPSs are lists of frequently asked questions that patients can take into a consultation. They are specified to the respective disease or condition the patient is in. Additionally, space is given for patients to take notes or record further questions. Their goal is to animate the patient to become a proactive customer by asking more questions during the consultation and therefore also gain more knowledge on their condition [32-33].

Arthur et al. (2017) tested the QPS in a palliative care setting. In total 100 patients and 12 physicians received the tool and were interviewed on their perception of its helpfulness. Overall, both patients and physicians had a positive connotation towards QPS. Most stated the tool was helpful for communicating with the physician (77\%), clear to understand (90\%) and they would use a similar tool in the future $(76 \%)$. Physicians perceived QPS as helpful for $68 \%$ of encounters and $73 \%$ stated it did not prolong the duration of the consultation. Acceptability and feasibility of this tool are rated very positively in this study. Additionally, patient anxiety was measured before and after consultation. Results indicated a significant decrease in patient anxiety after consultation. The results were, however, not compared to a control group, that did not receive QPS. This makes it difficult to link the usage of the tool to reduced anxiety, as patients could generally be less anxious after a consultation [34] (EL: IV).

The information obtained in Arthur et al. (2017) is supported by the study of Brown et al. (2001), which concludes that QPS, which are actively addressed by the physician during consultation, enhance information recall, reduce anxiety and shorten the length of the encounter. In order to reach these conclusions 318 patients with cancer, seeing their oncologists for the first time, were randomized to receive or not receive a QPS. The group that received the tool was again divided into patients, whose physicians would actively address the prompt sheet in the consultation and patients, whose physicians would not. The consultations were audio-taped and standardized questionnaires and interviews used, to gain information from the patients. The results indicated that patients with QPS asked more questions on prognosis and therefore received more information from their physician on the topic. If the tool was, however, not directly addressed by the physician, it had a negative impact: increasing patient anxiety after the encounter and prolonging consultation duration [35] (EL: II).

In 1999 the same author was already part of an intervention to promote questionasking behaviour in patients. The effectiveness of QPS was compared to coaching sessions exploring benefits and barriers to question-asking as well as rehearsal techniques. It was found that the QPS (addressed by the doctor) had a significantly greater effect on promoting patients to ask more questions, thus involving them in the consultation [36] (EL: II).

In contrast to this, a study by Butow et al. (1994) found that the QPS did generally not increase the number of questions asked, however questions on prognosis increased from $16 \%$ in the control group to $35 \%$ in the intervention group. In this randomized controlled trial 142 patients either received a QPS or a general paper informing them of available services in the institution [37] (EL: II). 
Further Patient Engagement Tools. Due to space limitations, the results of the other six patient engagement tools that were evaluated are not displayed in detail. Instead, a short summary is given in Table 4.

Table 4. Overview on effectiveness and feasibility of further patient engagement tools

\begin{tabular}{|l|c|c|l|}
\hline Tool & Effectivity & Feasibility & \multicolumn{1}{c|}{ Representative Sources } \\
\hline ACA & + & ++ & [38] EL: IV; [39] EL: IV; [40] EL: III \\
\hline BC/ WC & ++ & + & [41] EL: IV; [42] EL: IV \\
\hline Decision Box & 0 & + & [43] EL: IV \\
\hline PET & 0 & 0 & [44] EL: IV \\
\hline Patient Portal & 0 & + & [45] EL: III; [46] EL: I; [47] EL: II \\
\hline Roulette Wheel & + & 0 & [48] EL: IV; [49] EL: V \\
\hline
\end{tabular}

$+\quad$ Evidence for effectivity/ feasibility is present to a large extent

$+\quad$ Evidence for effectivity/ feasibility is generally present

0 Evidence is controversial or there are no sources available

- $\quad$ Evidence for effectivity/ feasibility is generally not present

-- $\quad$ Evidence for effectivity/ feasibility is not present to a large extent

Discussion of the Evidence on Patient Engagement Tools. When considering the results obtained, the effectivity and feasibility of the three patient engagement tools, Option Grid, Patient Diary, and QPS seems to generally be high. Especially for the Option Grid and the QPS both measures can be evaluated positively.

When summarizing the information obtained for the Option Grid, it can be concluded that there is no study displaying any negative impacts through the usage of Option Grids. Effectiveness, in terms of increasing SDM and patient engagement, was present in multiple studies. Feasibility is partially given, if the encounter is not prolonged through usage of the tool, which was measured and positively evaluated in one study. This is, however, surely dependent on the design of the Option Grid and training of the physicians. Acceptability of the tool seems to be very high, especially for patients.

Results for the effectiveness of Patient Diaries, in the sense of enhancing patient engagement, are controversial. Monitoring personal symptoms is already a form of engaging oneself with the individual health status. Self-reflection is fostered and decisions that need to be made with the physician are more informed, which can have a positive impact on SDM. Still, the two studies with the highest levels in the Oxford Scale of Evidence for Patient Diaries, both portrayed only marginal proof of benefits the tool may generate, so that a decisive conclusion is not possible without any further research on the topic. There is no evidence that Patient Diaries can have a negative impact. Feasibility seems to be present to a large extent. Acceptance for the Patient Diary was proven amongst a population of very young adults, in an online format of the tool. Feasibility is also fostered by the expert's opinion that retrieving the information from a Patient Diary is faster, therefore shortening the duration of consultations.

When summarizing the results of the studies found for the QPS, it can be concluded that acceptability and feasibility for the tool are high. Duration of the encounter (when used in the correct manner) is shortened through the QPS, which suggests a high 
feasibility. Helpfulness was also rated positively by patients and physicians. Effectiveness and therefore patient engagement is the extent to which patients are more involved in consultation and therefore ask more questions to gain an increased understanding of their condition. This is also the basis for SDM to take place. As shown in the studies, QPS are generally very effective for promoting question-asking behaviour. For this tool, it is noticeable that the records are comparably old. The most recent study from 2017, however, also reflects the positive results obtained in the other sources.

When considering the obtained results, it can be concluded that evidence for the effectivity and feasibility of Option Grids and QPS is present to a large extent. Evidence for these two criteria in Patient Diaries is at least given partially. It can therefore be derived that an enhanced usage of some patient engagement tools in practice has the potential to yield multiple benefits associated with patient engagement. Furthermore, the possibility that other patient engagement tools may also prove to be effective and feasible is conceivable.

\subsection{Representation and Utilization of Patient Engagement Tools in Patient Pathways}

Recommendation for Representation. In order to answer RQ2, two main areas of interest need to be discussed. First, it must be considered to which patient pathway elements the tools can be linked. Second, a meaningful representation of the tools in patient pathways, including variations for diverse characteristics, is necessary.

The Business Process Model and Notation (BPMN) is a domain-independent conceptual modelling language, commonly used as a visual representation of complex business processes in economy and industry. However, BPMN is also used for modelling care processes and is an established approach in health care practice [50-51]. There are healthcare-specific BPMN extensions for pathway modelling such as BPMN4CP [52]. For this reason, we choose BPMN4CP for patient pathway representation and patient engagement tool inclusion.

When considering the purpose of diverse patient engagement tools, it becomes clear that they are generally used to support specific tasks, e. g. communication or selfmanagement. Also, SDM is a task, which needs to be performed at some point in the process jointly by the physician and the patient. It is therefore clear, that SDM tools can be attached to this specific task, which will be prominent in all patient pathways, as these already have a focus on individual patient planning and management. What also supports the idea of attaching patient engagement tools to particular tasks, is that such tools always need to be introduced or handed over to the patient in some form. This means that someone must actively correspond with the patient about the tool. Tasks in patient pathways often already incorporate an interaction between the patient and a health care professional, through which the further integration of a tool at this point does not lead to additional efforts.

It should also be considered when, not only where, patient engagement tools can generally be used. Many cannot be used in every kind of pathway (depending on the condition) or with any type of patient. As an example, SDM tools can only be utilized 
for conditions in which there are multiple, reasonable different treatment options, these options are sensitive to preferences that patients may have (involve trade-offs) and the evidence for choosing one option over another must be uncertain [53]. Additionally, not all patients want to be involved in their care or in decision-making processes. Preferences can differ dramatically, meaning that a patient's personality must also be considered when deciding if and what kind of patient engagement tools to use [54]. Furthermore, a pathway should not be overloaded by diverse patient engagement tools. Some can be combined in a manner that makes sense, but for example using multiple different SDM tools for the same decision may only confuse the patient. Therefore, which tools fit best to the different workflows for conditions described through the pathways, needs to be thought through and tested individually.

The symbol proposed for the representation of patient engagement tools in patient pathways is depicted in Figure 1. It can be connected to the element in the patient pathway using a dotted line.

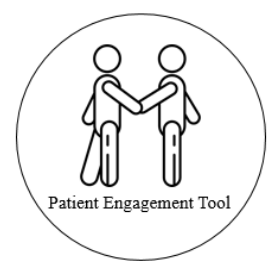

Figure 1. Symbol for patient engagement tool ${ }^{1}$

Different features that patient engagement tools possess can be depicted in the patient pathway through alternations of the patient engagement tool symbol. Depending on the type of patient engagement tool, the color of the symbol could change. SDM tools are depicted in orange, communication tools in yellow, self-management tools in green and tools for patient education are depicted in blue. If necessary, further color schemes could be added. Additionally, patient engagement tools in the form of documents (that could for example be printed, filled out together or handed out to the patient) should be distinguished from other types of tools by the form of a paper with a bent edge around the symbol. If the tool can be independently configured and therefore adapted to the individual patient through a health professional, it should be depicted through a screwdriver icon centrally placed at the top of the symbol.

\footnotetext{
${ }^{1}$ Icon made by Freepik from www.flaticon.com.
} 


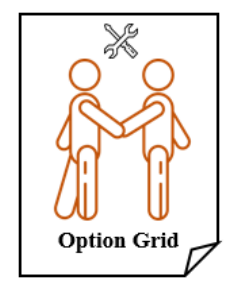

Figure 2. Engagement tool symbol for an adaptable Option Grid in the form of a document ${ }^{2}$

Additionally, further information to the user should be depicted in an extra view named "details", which opens when clicking on the patient engagement tool symbol. A practical example is given in the following section by applying the integration of QPSs in patient pathways to the oncology use case.

Application Example - Question Prompt Sheets. In order to demonstrate and test the recommendations for representation of patient engagement tools, a tool will be explored in the context of a colorectal cancer patient pathway for comprehensive cancer care networks. The QPS was chosen as an example, because evidence levels regarding its effectivity and feasibility are high and it is a tool that should fit into most pathways, unattached to the specific condition. The used patient pathway for colorectal cancer patients was developed as part of the large-scale European Joint Action iPAAC (Innovative Partnership for Action Against Cancer) ${ }^{3}$, aiming to develop and implement innovative approaches to cancer control.

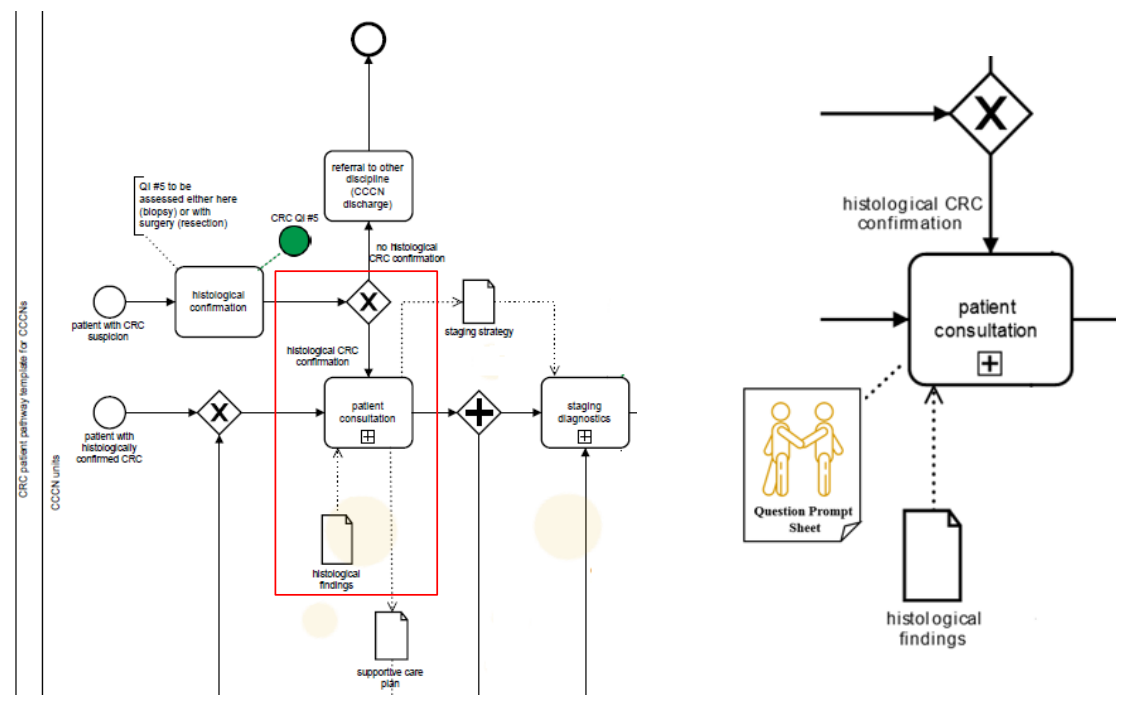

Figure 3. Representation of QPS in patient pathway for colorectal cancer (left: patient pathway model without QPS integration, right: detailed view on integrated QPS)

${ }^{2}$ Icons made by Freepik and Becris from www.flaticon.com.

${ }^{3}$ URL: https://www.ipaac.eu/ (accessed 25.08.2020) 
The QPS is used by the patient and the physician during consultation. Especially for complex diseases, such as cancer, several consultations take place. QPS are not a typical tool for SDM, as they do not focus on different treatment alternatives. Questions about these could be included, but the main aim is to promote general question asking behaviour (and therefore increase the amount of information obtained) by patients on their specific conditions. Especially during the first consultation after diagnosis patients often need a lot of information on their condition, due to which the integration of the tool during this task makes sense. When referring to the colorectal cancer patient pathway template, the QPS will be integrated at the initial "patient consultation" for patients that have a confirmed histological finding, which is depicted in Figure 3. This approach is supported by information in the study of Lambert et al. (2019), in which feedback from patients indicates that the QPS would be less valuable to them in review consultations [55]. For the QPS, a details-view with more instructions and further information on its usage can be retrieved (see Figure 4).

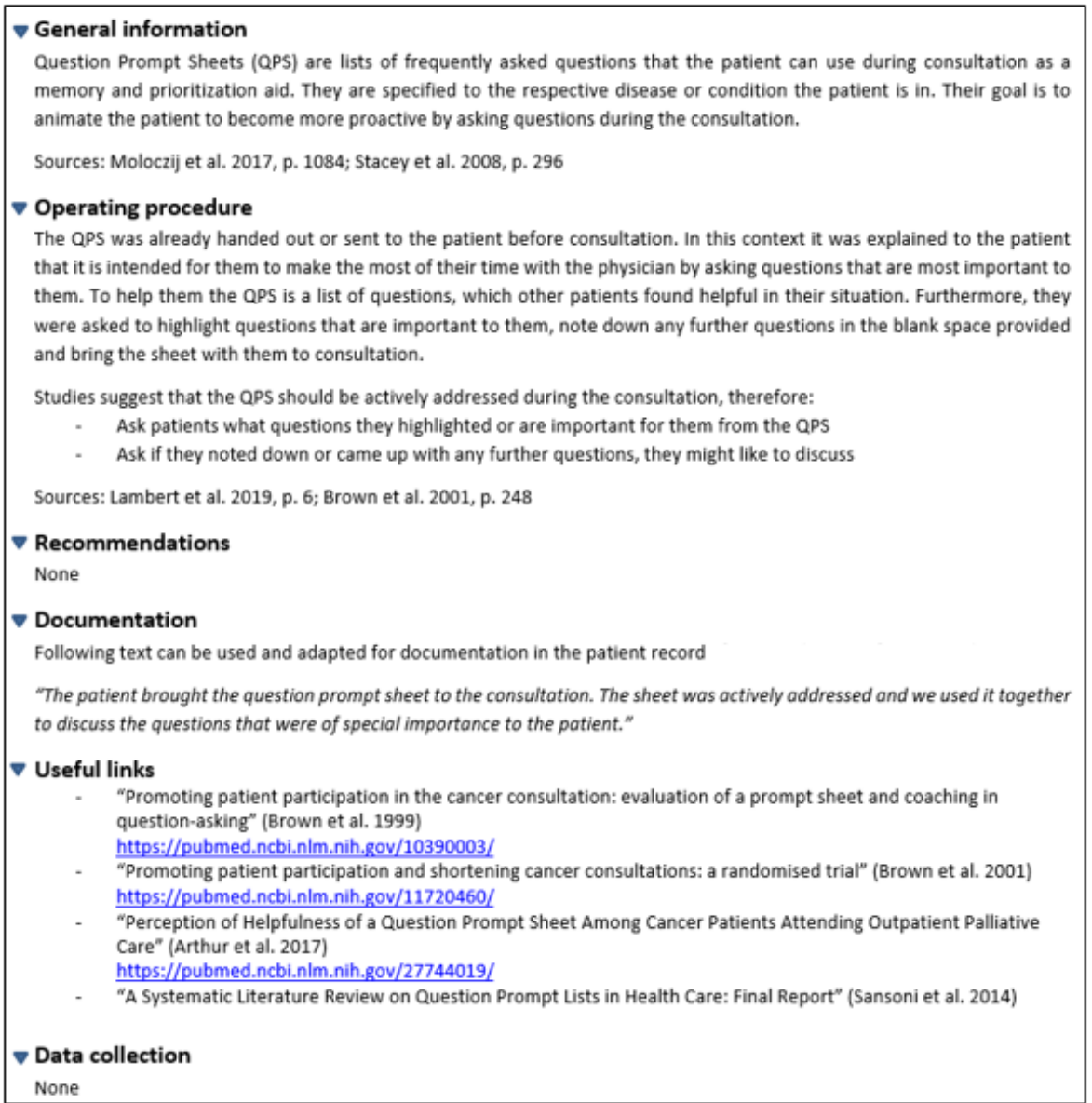

Figure 4. Example of a details-view for the QPS tool 


\section{Conclusion and Discussion}

Tools for patient engagement and SDM offer possibilities to enhance the active integration of patients into their own process of health care. Furthermore, their integration of specific tools in the form of concrete working instructions at specific pathway steps seems more practical and goal-oriented than a general proposal of working in a more patient-centred manner through a general explanation of the concepts of patient engagement. However, evidence for how well these tools foster patient engagement, how fluently they can be integrated into the process and how acceptable they are to patients and health care professionals needed to be explored. Therefore, a literature review was conducted to find out what evidence for the effectivity and feasibility of patient engagement tools exists (according to RQ1). Overall, it can be summarized that there is evidence for at least some patient engagement tools, including the Option Grid and the QPS. It must, however, also be considered that there is a lot of contradictory information. Furthermore, studies were often performed in very diverse setting, for example with different medical conditions, treatment options and participant groups. Also, the quality of the tool usage varied immensely depending on how and when it was put to action. Comparability between studies is therefore limited. It can also be concluded that research on patient engagement tools is only beginning to develop. Nearly all sources used for the review were published in the last five years.

Despite evidence for the effectivity and feasibility of some engagement tools, reports of their usage in practice remain rare. When embedding engagement tools in the already well-established concept of pathways, they may also gain more prominence and acceptance. This approach is a chance to close the gap between research and practice and therefore to eliminate inefficiencies through a suboptimal execution of health care services, which is the case if patients do not receive the chance of being involved. Only through patient engagement individual needs and preferences can be elicited and a better understanding of the condition by the patient leads to higher compliance and an enhanced communication. All these aspects ultimately lead to better health care outcomes and a higher quality of care. Therefore, we explored how patient engagement tools could be represented in patient pathways (according to RQ2). The proposed recommendations for representation where applied to a patient pathway for colorectal cancer using the QPS as an example. The goal was to demonstrate and test the recommendations given. After application, no further changes needed to be made to the initial representation format or to the general statement of where these tools can be connected to the pathway.

The results of this paper contribute to the mounting evidence that the usage of patient engagement tools in practice should be enhanced. The integration of these tools into patient pathways could be a substantial part of putting theory into practice. Several new research areas become prominent through these results. For example, the evaluation of patient engagement tools in practice, would be of interest to support the findings of this paper. The representation of patient engagement tools in patient pathways will be made possible by developing a BPMN extension to represent patient engagement tools in patient pathways. Furthermore, in the context of patient pathways, the active engagement of patients during their development could be exploited. These topics are of high interest to research and concrete plans for their realization are in progress. 


\section{Acknowledgements}

This article was partly funded by the European Union's Health Programme (20142020).

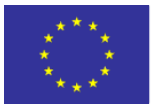

$$
\begin{aligned}
& \text { Co-funded by } \\
& \text { the Health Programme } \\
& \text { of the European Union }
\end{aligned}
$$

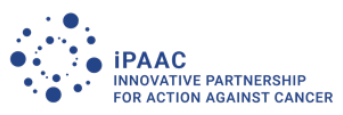

\section{References}

1. Vanhaecht, K.: The Impact of Clinical Pathways on the Organization of Care Processes. Technical report, Katholieke Universiteit Leuven (2007)

2. Küttner, T., Roeder, N.: Klinische Behandlungspfade. Deutscher Ärzte-Verlag, Köln (2007)

3. Gand, K., Schlieter, H.: Personalisation and Dynamisation of Care Pathways - Foundations and Conceptual Considerations. Research-in-Progress Report. 68 (2016)

4. The Value Collaborative: Putting Patients at the Center of the Clinical Pathway Debate. https://catalyst.phrma.org/putting-patients-at-the-center-of-the-clinical-pathways-debate (Accessed: 10.11.2020)

5. Richter, P., Schlieter, H.: Understanding Patient Pathways in the Context of Integrated Health Care Services - Implications from a Scoping Review. Proceedings of the $14^{\text {th }}$ International Conference of Wirtschaftsinformatik (2019)

6. Higgins, T., Larson, E., Schnall, R.: Unravelling the Meaning of Patient Engagement. A Concept Analysis. Patient Education and Counselling 100, 30-36 (2017)

7. Cerezo, P., Udina, J., Eulália et al.: Concepts and Measures of Patient Empowerment: A comprehensive Review. Revista da Escola de Enfermagem da USP (2016)

8. Doherr, H., Christalle, E., Kriston, L. et al.: Use of the 9-item Shared Decision Making Questionnaire in Intervention Studies: A Systematic Review. PLOS ONE 1-16 (2017)

9. Keddem, S., Agha, A., Long, J. et al.: Creating a Toolkit to Reduce Disparities in Patient Engagement. Medical Care 55, 59-69 (2017)

10. De Santis, M., Hervas, C., Weinman et al.: Patient Empowerment. National Center for Rare Diseases (2018)

11. Walker, D., Sieck, C., Menser et al.: Information Technology to Support Patient Engagement: Where Do We Stand and Where Can We Go? JAMA 24, 1088-1094 (2017)

12. Rowley, J., Slack, F.: Conducting a Literature Review. Manag Res News 27, 31-39 (2004)

13. Howick, J., Chalmers, I., Glasziou, P. et al.: The Oxford Levels of Evidence 2". https://www.cebm.net/index.aspx?o=5653 (Accessed: 09.10.2020)

14. Nijhuis, F., Elwyn, G., Bloem, B. et al.: Improving SDM in Advanced Parkinsons Disease: Protocol of a Mixed Methods Feasibility Study. Pilot and Feasbility Studies 4, 1-8 (2018)

15. Scalia, P., Elwyn, G., Barr, P. et al.: Exploring the Use of Option Grid Patient Decision Aids in a Sample of Clinics in Poland. ZEFQ 134, 1-8 (2018)

16. Elwyn, G., Frosch, D., Kobrin, S.: Implementing Shared-decision Making: Consider all the Consequences. Implementation Science 11, 1-10 (2016)

17. Elwyn, G., Rasmussen, J., Kinsey, K. et al.: On a Learning Curve for Shared-decision Making: Interviews with Clinicians Using the Knee Osteoarthritis Option Grid. Journal of Evaluation in Clinical Practice 24, 56-64 (2018) 
18. Kinsey, K., Firth, J., Elwyn, G. et al.: Patients Views on the Use of an Option Grid for Knee Osteoarthritis in Physiotherapy Clinical Encounters: An Interview Study. Health Expectations 20, 1302-1310 (2017)

19. Smith, S., Alvand, A., Locock, L. et al.: Partial or Total Knee Replacement? Identifying Patients Information Needs on Knee Replacement Surgery: A Qualitative Study to Inform a Decision Aid. Quality of Life Research 29, 999-1011 (2019)

20. Hahlweg, P., Witzel, I., Müller, V.: Adaptation and Qualitative Evaluation of Decision Aids in Breast Cancer Care. Archives of Gynecology and Obstetrics 299, 1141-1149 (2019)

21. Alam, S., Elwyn, G., Percac-Lima, S. et al.: Assessing the Acceptability and Feasibility of Encounter Decision Aids for Early Stage Breast Cancer Targeted at Underserved Patients. BMC Medical Informatics and Decision Making 16, 147 (2016)

22. Zisman-Ilani, J., Shern, D., Deegan, P. et al.: Continue, Adjust, or Stop Antipsychotic Medication: Developing and User Testing an Encounter Decision Aid for People with Firstepisode and Long-term Psychosis. BMC Psychiatry 18, 142 (2018)

23. Greenhalgh, T.: Option Grids: An Idea Whose Time Has Come? The British Journal of General Practice. The Journal of the Royal College of General Practitioners 63, 147 (2013)

24. Scalia, P., Elwyn, G., Barr, P. et al: Exploring the Use of Option Grid Patient Decision Aids in a Sample of Clinics in Poland. ZEFQ 134, 1-8 (2018)

25. Wright, S., Walsh, H., Ingley, K. et al.: Uptake of Self-management Strategies in a Heart Failure Management Programme. European Journal of Heart Failure 5, 371-380 (2003)

26. Cherenack, E., Wilson, P., Kreuzman, A. et al.: HIV/ AIDS Interventions: The Feasibility and Acceptability of Using Technology Based Daily diaries with HIV-infected Young Men Who have Sex with Men. AIDS and Behaviour 20, 1744-1753 (2016)

27. Park, L., Dracup, K., Whooley, M. et al.: Symptom Diary Use and Improved Survival for Patient with Heart Failure. Circulation. Heart Failure 10, (2017)

28. Hodge, B.: The Use of Symptom Diaries in Outpatient Care. FPM Journal 20, 24-28 (2013)

29. Himes, B., Weitzman, E.: Innovations in Health Information Technologies for Chronic Pulmonary Diseases. Respiratory Research 17, 1-7 (2016)

30. Schmidt, M., Eckardt, R., Scholtz, K. et al.: Patient Empowerment Improved Perioperative Quality of Care in Cancer Patients Aged 65 Years - A Randomized Controlled Trial. PloS One 10, e0137824 (2015)

31. Ullman, A., Aitken, L., Rattray, J. et al.: Diaries for Recovery from Critical Illness. Cochrane Database of Systematic Reviews 4, (2013)

32. Moloczij, N., Krishnasamy, M., Butow, P. et al.: Barriers and Facilitators to the Implementation of Audio-recordings and Question Prompt Lists in Cancer Care Consultations: A Qualitative Study. Patient Education and Counselling 6 (2017)

33. Stacey, D., Samant, R., Bennett, C.: Decision Making in Oncology: A Review of Patient Decision Aids to Support Patient Participation. ACS Journals 58, 293-304 (2008)

34. Arthur, J., Yennu, S., Zapata, K. et al.: Perception of Helpfulness of a QPS Among Cancer Patients Attending Outpatient Palliative Care. JPSM Journal 53, 124-130 (2017)

35. Brown, R., Butow, P., Dunn, S. et al.: Promoting Patient Participation and Shortening Cancer Consultation: A Randomised Trial. British Journal of Cancer 85, 1273-1279 (2001)

36. Brown, R., Butow, P., Boyer, M. et al.: Promoting Patient Participation in the Cancer Consultation: Evaluation of a Prompt Sheet and Coaching in Question-Asking. British Journal of Cancer 80, 242-248 (1999)

37. Butow, P., Dunn, S., Tattersall, M. et al.: Patient Participation in the Cancer Consultation: Evaluation of a QPS. Annals of Oncology. ESMO Open 5, 199-204 (1994)

38. Streufert, B., Reed, S., Orlando, L. et al.: Understanding preferences for treatment after hypothetical fit-time anterior shoulder dislocation. AOSSM 5, (2017) 
39. Dunlea, R., Lenert, L.: Understanding patients' preferences for referrals to specialists for an asymptomatic condition. SAGE Journals 35, 691-702 (2015)

40. Hess, L., Litwiller, A., Byron, J. et al.: Preference elicitation tool for abnormal uterine bleeding treatment: a randomized controlled trial. The Patient 8, 217-227 (2015)

41. Chesney, T., Deveon, K.: Training surgical residents to use a framework to promote shared decision-making for patients with poor prognosis experiencing surgical emergencies. Canadian Journal of Surgery 61, 114-120 (2018)

42. Taylor, L., Nabozny, M., Steffens, N. et al.: A framework to improve surgeon comm. in high-stakes surgical decisions: best case/ worst case. JAMA Surgery 152, 531-538 (2017)

43. Giguere, A., Labrecque, M., Haynes, B.: Evidence summaries to prepare clinicians for shared decision-making with patients. Implementation Science, (2014)

44. Lastinger, A., Gomez, K., Manegold, E. et al.: Use of a patient empowerment tool for hand hygiene. American Journal of Infection Control, (2017)

45. Dendere, R., Slade, C., Burton-Jones, A. et al.: Patient portals facilitating engagement with inpatient electronic medical records: a systematic review. JMIR 21, e12779 (2019)

46. Ammenwerth, E., Schnell-Inderst, P., Hoerbst, A.: The impact of electronic patient portals on patient care: a systematic review of controlled trials. JMIR 14, e162 (2012)

47. Fiks, A., Mayne, S., Karavite, D. et al.: Parent-reported outcomes of a shared decisionmaking portal in asthma: a practice-based RCT. Pediatrics 135, e965-e973 (2015)

48. Scalia, P., O’Malley, J., Durnd, M. et al.: Presenting time-based risks of stroke and death for patients facing carotid stenosis treatment options: Patients prefer pie charts over icon arrays. Patient education and counselling 102, (2019)

49. Hoffmann, J., Wilkes, M., Day, F.: The roulette wheel: An aid to informed decision making. PLoS Medicine 3, (2006)

50. Scheuerlein, H., Rauchfuss, F., Dittmar, Y. et al.: New methods for clinical pathwaysBPMN and t.BPM. Langenbecks Archives of Surgery 397, 755-761 (2012)

51. Zerbato, F., Oliboni, B., Combi, C. et al.: BPMN-Based Representation and Comparison of Clinical Pathways for Catheter-Related Bloodstream Infections. International Conference on Healthcare Informatics, 346-355 (2015)

52. Braun, R., Schlieter, H., Burwitz, M. et al.: BPMN4CP Revised - Extending BPMN for Multi-perspective Modeling of Clinical Pathways. Proceedings of the 49th Hawaii International Conference on System Sciences (HICSS), 3249-3258 (2016)

53. National Health Service England: When and where is shared decision making appropriate? https://www.england.nhs.uk/shared-decision-making/when-and-where-is-shared-decisionmaking-appropriate/ (Accessed: 10.11.2020)

54. Fredriksson, M., Eriksson, M., Tritter, J.: Who wants to be involved in health care decisions? Comparing preferences for individual and collective involvement in England and Sweden. BMC Public Health 18, 18 (2018)

55. Lambert, K., Lau, T., Davidson, S. et al.: Development and Results on the Feasibility of a Renal Diet Specific QPS for Use in Nephrology Clinics. BMC Neph. 20, 48 (2019) 